\title{
Tactus $\neq$ Tempo: Some Dissociations Between Attentional Focus, Motor Behavior, and Tempo Judgment
}

\author{
JUSTIN LONDON \\ Carleton College
}

\begin{abstract}
Three experiments explored the relationships between surface rhythmic activity, tactus or beat rate, attentional focus, sensorimotor synchronization (tapping), and tempo perception. All involved a rhythmic standard followed by a comparison; the experimental task was a judgment of "slower, same, or faster." In Experiment 1 participants simply judged relative speed; they focused on the beat level in Experiment 2, and they tapped along as they made their judgments in Experiment 3. In all three experiments judgments were highly accurate $(89-97 \%$ correct, relative to beat-level inter-onset interval) when the standard-comparison involved the same pattern/same tempo, and performed similarly for the same pattern at different tempos $(80-83 \%$ correct). Performance degraded significantly in other contexts, especially for different patterns at the same tempo. A main effect for pattern (two levels: same vs. different) and a pattern $\mathrm{x}$ tempo interaction were observed in all three experiments; a main effect for tempo (collapsed to two levels: same vs. different) occurred only in Experiment 1. Analysis of a subset of the experimental conditions indicated that surface activity was of greater salience than the beat level in some contexts. Tapping along (Experiment 3) did not improve overall performance any more than simply focusing on the tactus level (Experiment 2), and a possible biasing effect of tapping rate on tempo judgment was observed. Thus there is an apparent dissociation between tactus rate, attentional focus, tapping behavior and tempo judgment. This suggests that our perception of musical speed or tempo is more than simple apprehension of the tactus rate.
\end{abstract}

Submitted 2010 August 18; accepted 2010 November 23.

KEYWORDS: rhythm, meter, tempo, tapping, beat, tactus, attention

\section{INTRODUCTION AND BACKGROUND}

EVER since Johann Nepomuk Mälzel patented and began manufacture of his mechanical metronome in 1815, musicians have made ready use of metronome markings as an accurate and convenient way of indicating the proper tempo (Thayer \& Harvey, 2010). Indeed, the "MM" still seen in many scores is an abbreviation for "Mälzel's Metronome." Metronome markings are now ubiquitous, with a common rhythmic value - typically a quarter note in simple meters and a dotted quarter in compound metersindexed to a number of ticks per minute. As a practical matter metronome markings work wonderfully well; moreover, it allows composers to give specific tempo directions to a degree that was unprecedented before the early 19th century. But do metronome markings really give an accurate indication of our subjective sense of musical speed? Figure 1 illustrates the problem; it gives one of the standard-comparison pairs used in the experiment (click here for a link to an audio version of Figure 1).

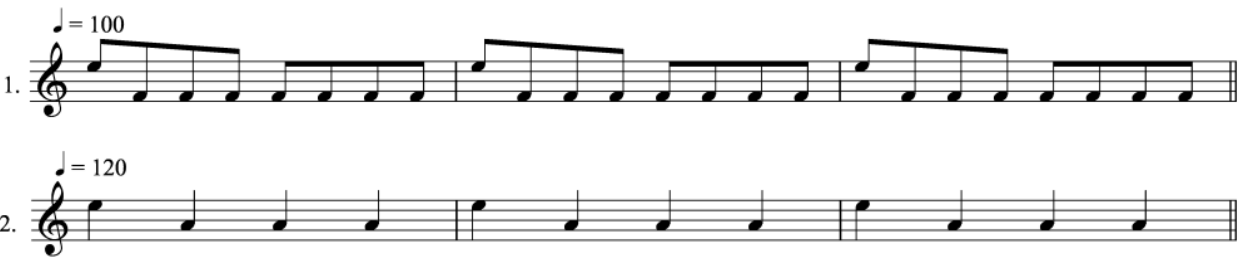

Figure 1. Sample standard-comparison stimulus pair. 
Which is faster, standard or comparison? The standard involves a series of wood-block articulations with an inter-onset interval (IOI) of $300 \mathrm{~ms}$, organized into a larger cycle of 8 ticks (total 2400 $\mathrm{ms}$ ). The comparison involves a series of four $500 \mathrm{~ms}$ IOIs, creating a larger cycle $2000 \mathrm{~ms}$ in duration. In the standard the $300 \mathrm{~ms}$ IOI is too fast to be heard as the beat or pulse (Fraisse, 1982; Clarke, 1999). Moreover, the $300 \mathrm{~ms}$ rate also affords the perception of a $600 \mathrm{~ms}$ periodicity, and according to pulsefinding research (Eck, 2001; Parncutt, 1994), the $600 \mathrm{~ms}$ period should be maximally salient. In the comparison, while its overall cycle duration is shorter (2000 vs. $2400 \mathrm{~ms}$ ), its surface IOIs are longer (500 ms vs. $300 \mathrm{~ms}$ ). Yet the comparison's $500 \mathrm{~ms}$ IOI is likely to be heard as the pulse (i.e., and not the latent $1000 \mathrm{~ms}$ period). Thus if relative speed is primarily dependent on pulse rate, then the comparison should be deemed faster than the standard. But if our sense of musical speed is cued more by surface IOIs/event density, then the comparison should be deemed slower than the standard. Thus there is a confound between pulse rates and surface activity in this standard/comparison pair.

There are actually two questions regarding metronome marks and our sense of the music's speed: (1) Do metronome markings indicate the beat rate? (2) Even if they do, is the beat rate itself a transparent measure of musical speed ${ }^{[1]}$ Regarding the former, while metronome markings directly indicate the rate of one layer of rhythmic activity, in most musical contexts other layers of rhythmic activity are present, both within individual parts as well as among the various instruments or voices in the musical texture. Thus at a metronome marking of 75 BPM (800 ms IOI), duplet 8th notes go twice as fast (400 ms IOI), half notes at half the rate (1600 ms IOI), and so forth. It is not clear, however, whether the perceived pulse of the music in this case would be 75 or 150 BPM (Collyer, Broadbent, et al.,1994; Large, Fink, \& Kelso, 2002; Martens, 2005; McKinney \& Moelants, 2006). The situation is even more complicated when one recognizes that the beat may be (and often is) divided not only into duplets, but also triplets, quadruplets, and/or quintuplets or sextuplets, and the subdivision of the beat may change repeatedly over the course of a musical passage.

Moreover, London $(2001,2004)$ has noted that the same rhythmic surface can give rise to alternative metrical construals on the part of the listener (cf. the discussion of "metric malleability" in London, 2004, pp. 48-50). Thus two rhythmic patterns with the same number of onsets and same interonset intervals on the surface (i.e., equal event density) played at the same metronome marking may nonetheless have different endogenous metrical organizations, with different component periodicities and nesting relationships. In such cases the beat rate itself may often be ambiguous, and if so then such patterns may not be judged to be equivalent in tempo.

Nonetheless, BPM measurements are often regarded as a reasonably transparent measure of musical speed (Jones \& Boltz, 1989; Moelants 2006; Parncutt, 1994; Quinn \& Watt, 2006; van Noorden \& Moelants, 1999), as are tapping behaviors yoked to the beat or pulse rate (Clynes \& Walker, 1982; Drake, Penel \& Bigand, 2000; Martens, 2005; McKinney \& Moelants, 2006; Snyder \& Krumhansl, 2001). Yet a number of authors have noted that beat rate and/or tapping rate can be dissociated from perceived tempo (Epstein, 1979, 1995; Drake, Gros \& Penel, 1999). That is, if the beat rate is the periodicity which best affords some form of bodily synchronization with the rhythmic stimulus (e.g., foot tapping, marching, arm swaying etc.), that optimal rate of synchronization may or may not directly correspond to our tempo percept. More plainly, we may tap slowly to quick music, and vice-versa. Our sense of musical speed involves a more complex grasp of musical structure than simply finding the pulse. Epstein (1995, p. 99) perhaps put it best:

Tempo is a consequence of the sum of all factors within a piece--the overall sense of a work's themes, rhythms, articulations, "breathing," motion, harmonic progressions, tonal movement, contrapuntal activity. . . Tempo ... is a reduction of this complex Gestalt into the element of speed per se, a speed that allows the overall, integrated bundle of musical elements to flow with a rightful sense.

The current study attempts to unpack Epstein's "integrated bundle of musical elements" by considering the effect of metrical structure-specifically, the presence or absence of various rhythmic layers both above and below the perceived tactus - on our perceived sense of speed. By metrical structure I mean the sense of entrainment between listeners and the music. This entrainment involves not only beats, but also beat subdivisions as well as higher level beat cycles, that is, the number and relative rates of hierarchically-nested periodicities that a rhythmic pattern embodies (for more details on meter-asentrainment, see London 2004). 
This study is grounded in a number of hypotheses:

1. Differences in metrical structure will affect subjective judgments of tempo-that is, even if two passages involve the same beat rate (i.e., a common periodicity in a middle range) the presence or absence of additional periodicities above and/or below the tactus will affect our sense of tempo and motion.

2. Subdivisions of the tactus (i.e., surface event density) will be more salient than the beat rate itself in making tempo judgments.

3. Given the cardinal role the tactus level has in anchoring our temporal perception (cf. Jones \& Boltz, 1989 , on the tactus as the "referent level" for temporal attending), focusing on the tactus level will improve the accuracy/inter-participant agreement of tempo judgments.

4. Given hypothesis (3), tapping along with the stimuli at the tactus rate will further improve consistency of tempo judgments.

Hypotheses 1 and 2 are expressions of skepticism re beat rate as the primary cue for tempo judgment, while Hypotheses 3 and 4 nonetheless acknowledge the contribution of the beat rate to our judgments of tempo.

\section{METHODS}

\section{Design and Experimental Task}

Three experiments were conducted using a set of stimuli which systematically varied the interactions between the rate of surface activity and the number of and depth of higher levels of rhythmic/metrical organization. In all stimuli the tactus level was unambiguously present. A within-subjects design was employed in all three experiments. A rhythmic pattern, exemplifying a basic metrical configuration was presented as a standard, followed by a comparison pattern. The primary experimental task was to judge if the comparison was either slower, the same, or faster than the standard. In the third experiment, participants also tapped along with the standard and comparison.

There were four stimulus pairing conditions, of which the participants were advised:

- Same Pattern @ Same Tempo (SPST)

- Same Pattern @ Different Tempi (SPDT)

- Different Pattern @ Same Tempo (DPST)

- Different Pattern @ Different Tempi (DPDT)

Tempo (same or different) was operationalized as the rate of the tactus level (i.e., what would be the typical metronome marking for each stimulus component). This by itself yields a $2 \times 2$ factorial design. As the "different" tempo response could be either slower or faster, the analysis may be expanded to a $2 \times 3$ factorial: 2 levels for patterns (same vs. different) and 3 levels of tempo (slower, the same, or faster). Subsets of the data (direction of the tempo change, number and relative depth of rhythmic layers, and so forth) afforded further factorial analysis as detailed in the results section below.

The first two experiments differed only with respect to the instructions given to the participants. In Experiment 1 participants were simply asked to make a judgment about the speed of the comparison in relation to the standard, while in Experiment 2 they were instructed to focus on the beat rate to make their tempo comparisons. In Experiment 1 if participants asked if they were to "listen to or for the beat," as sometimes happened if the participant had a high degree of musical training, they were enjoined not to count or attend to beats but simply to make a judgment based on their overall impression of the stimuli's relative speeds. In Experiment 2, subjects were asked if they understood what was meant by "the beat," and if unsure, a brief tutorial demonstration was given. In both Experiments 1 and 2 participants were enjoined not to tap along or employ other overt movements as they listened. 
In Experiment 3 participants were instructed to tap along with the beat or pulse of the music and a brief demonstration/tutorial (using sample melodies not used in the experiment) was given. Participants were told not to tap immediately, but to listen for a few beats before tapping to the standard and then do the same with the comparison. They were told not to continue tapping through the pause between standard and comparison, but it was OK to tap once or twice after each pattern finished.

In all three experiments, participants were told to listen to each stimulus pair to its completion before making their tempo judgments; in Experiment 3 they were also enjoined to tap through to the end of the comparison before making their tempo judgments.

\section{Stimuli}

Figure 2 lists the seven (7) basic patterns that were used as both standards and comparisons; the different pitches in the musical notation indicate the different wood-block sounds that were used to differentiate the metrical layers in the various stimuli. While there were pitch differences among the wood-block sounds, they were not "melodic" patterns in the usual sense.
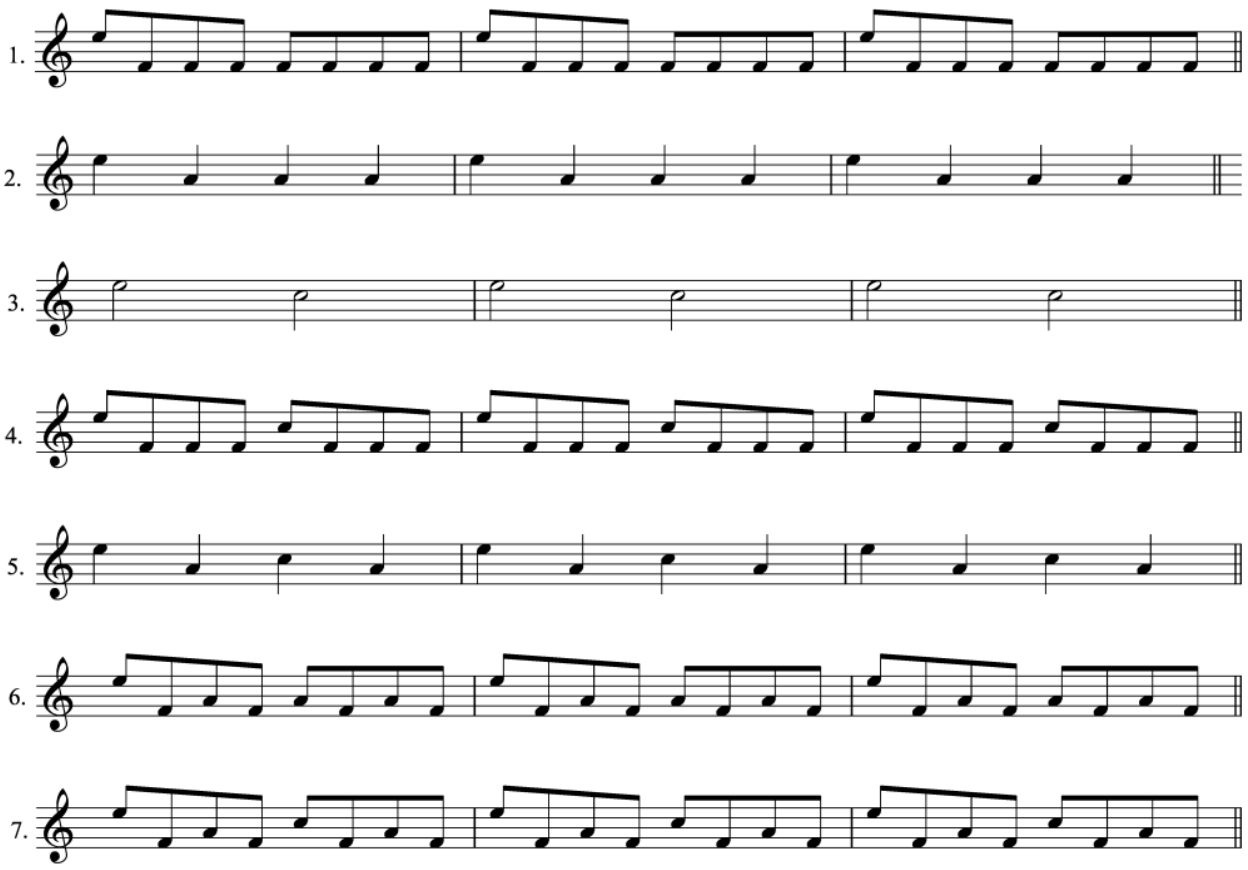

Figure 2. Set of all stimulus patterns used in all three experiments; patterns 1-3 articulate two layers of metrical structure, patterns 4-6 three layers, and pattern 7 four layers.

While it is obvious in Figure 2 that some stimuli involved constant eighth notes (patterns 1, 4, 6, and 7), others quarter notes (2 and 5), and one only half notes (3), note that each pattern may or may not articulate additional layers of metrical activity. Stimuli were presented at either 100 or 120 BPM, which, depending upon the stimulus pattern, involved component IOIs of 2400/1200/600/300 and 2000/1000/500/250 ms, respectively. As can be seen in Figure 2, every pattern involved the longest period (2400 ms or $2000 \mathrm{~ms}$ ) plus at least one other component periodicity. Stimuli with an overall cycle duration of $2000 \mathrm{~ms}$, with an implied or explicit beat rate of $500 \mathrm{~ms}$ IOI were coded as "fast," while stimuli with a cycle duration of $2400 \mathrm{~ms}$ (i.e., $600 \mathrm{~ms}$ IOI for the beat level) were coded as "slow."

All stimuli were in 4/4 meter and the tactus/beat level was clear; pulse finding was not deemed to be a problem in any of these stimuli. Participants were presented will all possible pairs in all possible orders and at all tempo permutations (same, slow-fast, or fast-slow), yielding a total of 154 stimuli. Each participant heard each stimulus once. Between the standard and the comparison was a silent interval of between 3-5 seconds; this interval was deemed long enough to prevent a direct carry-over of the beat rate from the standard, while short enough to afford good tempo comparison. The length of the inter-stimulus 
interval was controlled to ensure that the onset of the comparison was out of phase with the beat and downbeat periods of the standard. Stimuli were arranged in quasi-random order (adjusted by hand to avoid clusters involving the same standard or comparison) and placed into two blocks, with block presentation counter-balanced among participants.

\section{Apparatus}

Stimuli were sequenced using Digital Performer 5.13 as standard MIDI files, on an Apple iMAC computer running OSX 10.5.8 with 2 MB Ram. Sounds were produced using the built-in Apple Software Synthesizer sound module using the woodblock percussion sound patch (GM \#116). Stimuli were presented in a quiet seminar room via over-the-ear headphones which afforded additional sound isolation (either Beyerdynamic DT 770 headphones in Experiments 1 and 2 or Sennheiser HD280pro headphones in Experiment 3). In Experiments 1 and 2 participants cued successive stimuli by clicking the appropriate button on the Digital Performer interface (N.B., the screen was arranged such that participants could not see the tempo, beat count, or clock readouts on the interface window). In Experiment 3 stimuli were cued by the experimenter when prompted by the participant after completion of the previous trial.

Response judgments in all three experiments regarding the tempos of the standard/comparison were collected via a tally sheet, and then coded and entered into Excel and SPSS for analysis. In Experiment 3, tapping data was collected via a Roland SPD6 drum pad synched to the Digital Performer set to the "bare hand" tapping sensitivity. Participants were able to place the drum pad in a comfortable position and were free to use any mode of tapping (finger, wrist, or forearm) so long as it produced an adequate signal level. Data regarding tapping mode and accuracy were hand-coded into an Excel spreadsheet using the Digital Performer "quick scribe" feature which produced a quantized output in music notation, aligned relative to the stimulus pair in each trial. This allowed us to determine if participants were able to successfully perform the tapping task and to determine if their tapping behavior changed within and/or between trials.

\section{Participants}

Table 1 gives a summary of the participants in each experiment:

\begin{tabular}{|l|l|l|l|l|}
\hline & $\begin{array}{l}\text { Number of } \\
\text { Participants }\end{array}$ & $\begin{array}{l}\text { Male/ } \\
\text { Female }\end{array}$ & $\begin{array}{l}\text { Training } \\
\mathbf{< 5 / 5 - 1 0 / > 1 0 ~ y r s . ~}\end{array}$ & $\begin{array}{l}\text { Age Data: } \\
\text { Median, Max-Min }\end{array}$ \\
\hline Exp 1 & $\mathrm{n}=24$ & $10 / 14$ & $6 / 7 / 11$ & $20 \mathrm{yrs}, 22-18$ yrs \\
\hline Exp 2 & $\mathrm{n}=12$ & $7 / 5$ & $2 / 4 / 6$ & $20 \mathrm{yrs}, 64-15 \mathrm{yrs}$ \\
\hline Exp 3 & $\mathrm{n}=19$ & $11 / 8$ & $6 / 7 / 6$ & $21 \mathrm{yrs}, 59-19 \mathrm{yrs}$ \\
\hline
\end{tabular}

Table 1. Summary of participant numbers, gender, musical training, and age in all three experiments.

Musical background/training levels were set at $<5 \mathrm{yrs}, 5-10 \mathrm{yrs}$, $>10 \mathrm{yrs}$ of formal training on an instrument or in voice, based on the distributions in the participant population. Participants in Experiment 1 were recruited from the author's Spring 2008 Introduction to the Perception and Cognition of Music class; participation in the experiment was one means of fulfilling a course requirement. Participants in Experiments 2 and 3 were recruited from the Carleton College and Northfield Community; they were not paid for their participation. There was no overlap between participant pools in the three experiments. In all three experiments subject recruitment and experimental procedure were approved by the Carleton College IRB and informed consent was obtained from all participants. 


\section{RESULTS}

\section{Overall Effects of Pattern and Beat Rate}

No effects of order or gender were found; in Experiment 1 some effect of training was apparent, but did not reach statistical significance; the effect of level of training was non-significant in Experiments 2 and 3. In all three experiments no significant differences in the "different" response categories in terms of slow-fast versus fast-slow were observed (i.e., no effect of presentation order) and the results were pooled for a $2 \times 2$ factorial analysis (pattern: same versus different; tempo: same versus different). Main results of all three experiments are given in Figure 3.

In Experiment 1 main effects were observed for pattern, $\mathrm{F}(1,23)=237.04, p<.001, \eta^{2}=.912$, and tempo, $\mathrm{F}(1,23)=7.21, p=.013, \eta^{2}=.239$ (Two-way repeated measures ANOVAs were used for analysis of main effects in all three experiments). There was also a significant interaction between pattern and tempo, $\mathrm{F}(1,23)=37.72, p<.001, \eta^{2}=.621$. Differences between trial conditions were significant in all cases (Wilcoxon Signed Ranks Test). ${ }^{[2]}$ When the pattern and tempo are both the same (SPST condition), judgments are near-perfect and very good in the same pattern/different tempo (SPDT) condition (see Table 2 for exact results in all three experiments). When the patterns are different, performance is substantially worse, though still above chance-performance for different patterns at the same tempo (DPST) falls to $40.77 \%$ correct, and performance for different patterns at different tempos (DPDT) is at $63.0 \%$ correct. Interestingly, the lowest scores occurred in the DPST condition.

In Experiment 2 a significant main effect was observed for pattern, $\mathrm{F}(1,11)=130.74, p<.001, \eta^{2}$ $=.922$, and there was a significant interaction between pattern and tempo, $\mathrm{F}(1,11)=5.92, p<.033, \eta^{2}=$ .350. However, there was not a significant main effect for tempo. Differences between trial conditions were not significant between DPDT/DPST, but were significant in all other cases (Wilcoxon Signed Ranks Test). The difference between the SPDT and SPST conditions increased in Experiment 2, whereas that between DPDT and DPST decreased, relative to Experiment 1. Recall that the difference between Experiments 1 and 2 was one of participant focus: in the first experiment participants simply judged the "speed" of each stimulus, while in the second they were told to do so while focusing on the beat rate. However, no significant differences in performance were found between each of the four stimulus conditions between the two experiments (Mann-Whitney U Test), though the difference for the DPDT condition approached significance $(\mathrm{U}=92.000, \mathrm{Z}=-1.746, p=.081)$.

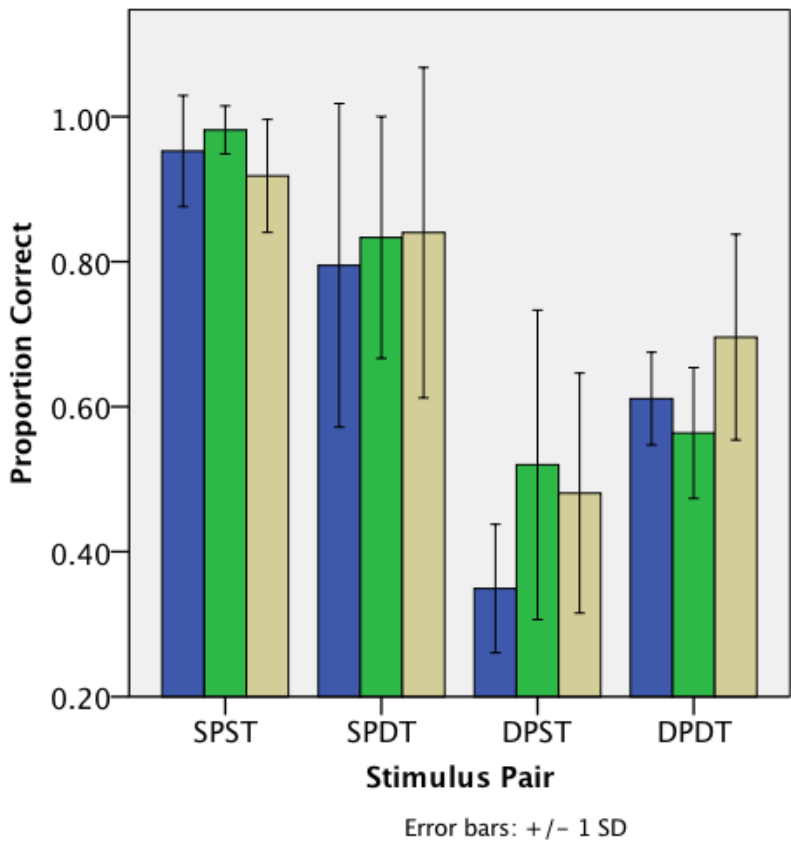

Experiment 1

Experiment 2

Experiment 3

Figure 3. Summary of main results from Experiments 1, 2, and 3. 
As in Experiment 2, in Experiment 3 a significant main effect was observed for pattern, $\mathrm{F}(1,18)=$ $29.309, p<.001, \eta^{2}=.620$, and there was a significant interaction between pattern and tempo, $\mathrm{F}(1,18)=$ $11.03, p=.004, \eta^{2}=.380$. As in Experiment 2 , there was not a significant main effect for tempo, $(\mathrm{F}(1,18)$ $=0.452, p=.510$ ). All conditions are significantly different from each other (Wilcoxon Signed Ranks Test), save for SPDT vs. SPST which was nearly significant $(Z=-1.863, p=.062)$.

Figure 3 gives the combined results of all three experiments, and Table 2 gives the values for each result. In the DPDT condition Experiment 3 is significantly different from Experiment 2 (Mann-Whitney U $=60.0, \mathrm{Z}=-2.191, p=.028)$ and also higher than in Experiment 1 . In the DPST condition, Experiment 3 is significantly different from Experiment 1 (Mann-Whitney $\mathrm{U}=218, \mathrm{Z}=-2.230 ; p=.026$ ), and in the SPST condition, Experiment 3 is significantly different from Experiment 2 (Mann-Whitney $U=61.5, Z=-2.256$; $p=.024)$. Thus relative to Experiments 1 and 2, tapping in Experiment 3 did help in the DPDT condition. In the DPST condition tapping helped no more than simply focusing on the beat rate (Experiments $2 \& 3$ vs. Experiment 1), and indeed, in the SPST condition tapping made performance slightly worse than simply focusing on the beat rate (Experiment 3 vs. Experiment 2). Note that in all three experiments, the DPST condition was the most difficult.

\begin{tabular}{|l|c|c|c|c|c|c|c|c|}
\hline & \multicolumn{2}{|c|}{ SPST } & \multicolumn{2}{c|}{ SPDT } & \multicolumn{2}{c|}{ DPST } & \multicolumn{2}{c|}{ DPDT } \\
\cline { 2 - 9 } & Mean & SDev & Mean & SDev & Mean & SDev & Mean & SDev \\
\hline Exp 1 & 95.24 & 8.07 & 86.54 & 17.46 & 40.77 & 17.46 & 63.00 & 9.12 \\
\hline Exp 2 & 98.17 & 3.33 & 83.25 & 16.59 & 52.00 & 21.33 & 56.33 & 9.01 \\
\hline Exp 3 & 88.84 & 13.12 & 79.68 & 25.05 & 48.42 & 15.27 & 68.21 & 15.01 \\
\hline Average & $\mathbf{9 4 . 0 8}$ & $\mathbf{8 . 1 7}$ & $\mathbf{8 3 . 1 6}$ & $\mathbf{1 9 . 7 0}$ & $\mathbf{4 7 . 0 6}$ & $\mathbf{1 8 . 0 2}$ & $\mathbf{6 2 . 5 1}$ & $\mathbf{1 1 . 0 5}$ \\
\hline
\end{tabular}

Table 2. Comparison of participant scores and standard deviations for each condition in all three experiments.

\section{DPDT Subset Analyses}

To gain a better sense of the interaction between surface vs. tactus periodicities, a subset of the DPDT data from each experiment was examined, specifically when the standard was slower than the comparison. In this context the "correct" answer should in all cases be "Faster." Figure 4 gives detailed results from the DPDT condition in all three experiments, and Table 3 gives pairwise comparisons for the responses in each experiment. The labels on the abscissa refer to the surface activity of the comparison (the target) versus the standard. So, "Surface Slower" is a case like that given in the audio demonstration-e.g., a 2400/300 ms standard followed by a 2000/500 ms comparison, such that the comparison's surface rate produces a cue that is mismatched to the relative beat rates. "Surface Commensurate" refers to trials where the standard and comparison have analogous cues-e.g., a 2400/300 ms standard followed by a 2000/250 ms comparison. The "Surface Faster" refers to those trials where the comparison was faster and involved nonanalogous rhythms - e.g., a 2400/600 ms standard followed by a 2000/250 ms comparison; in the surface faster condition the increased surface rate reinforces the increased tactus rate. Lastly, the SPDT results are given as a benchmark. It can be seen that in "Surface Slower" cases, most participants got the "wrong" answer, while in other cases they mostly got the "right" answer. The "Surface Slower" condition was significantly different from the other two conditions and the SPDT benchmark, while there were no significant differences among the other three, as shown in Table 3. In Experiment 2, when focusing on the beat rate, the differences between Slower, Same, and Faster are less significant (and in the case of Slower vs. Faster, non-significant), and performance relative to the benchmark degrades. Similar results were obtained in Experiment 3. 


\begin{tabular}{|l|l|c|c|c|c|c|c|}
\hline & & Slower/Same & Slower/Faster & Same/Faster & Slower/SPDT & Same/SPDT & Faster/SPDT \\
\hline \multirow{2}{*}{ Exp. 1 } & $\mathrm{Z}$ & -4.294 & -4.162 & -1.181 & -4.291 & -.187 & -1.545 \\
\cline { 2 - 8 } & Sig. & .000 & .000 & .238 & .000 & .852 & .122 \\
\hline \multirow{2}{*}{ Exp. 2 } & $\mathrm{Z}$ & -2.197 & -1.413 & -1.020 & -2.981 & -2.591 & -2.040 \\
\cline { 2 - 8 } & Sig. & .028 & .158 & .308 & .003 & .010 & .041 \\
\hline \multirow{2}{*}{ Exp. 3 } & $\mathrm{Z}$ & -3.012 & -2.407 & .000 & -3.042 & -.371 & -.807 \\
\cline { 2 - 8 } & Sig. & .003 & .016 & 1.000 & .002 & .711 & .420 \\
\hline
\end{tabular}

Table 3. Z-scores and $p$ values from a Wilcoxon Signed Ranks Test of paired comparisons for subsets of the DPDT Slow-Fast condition in all three experiments.

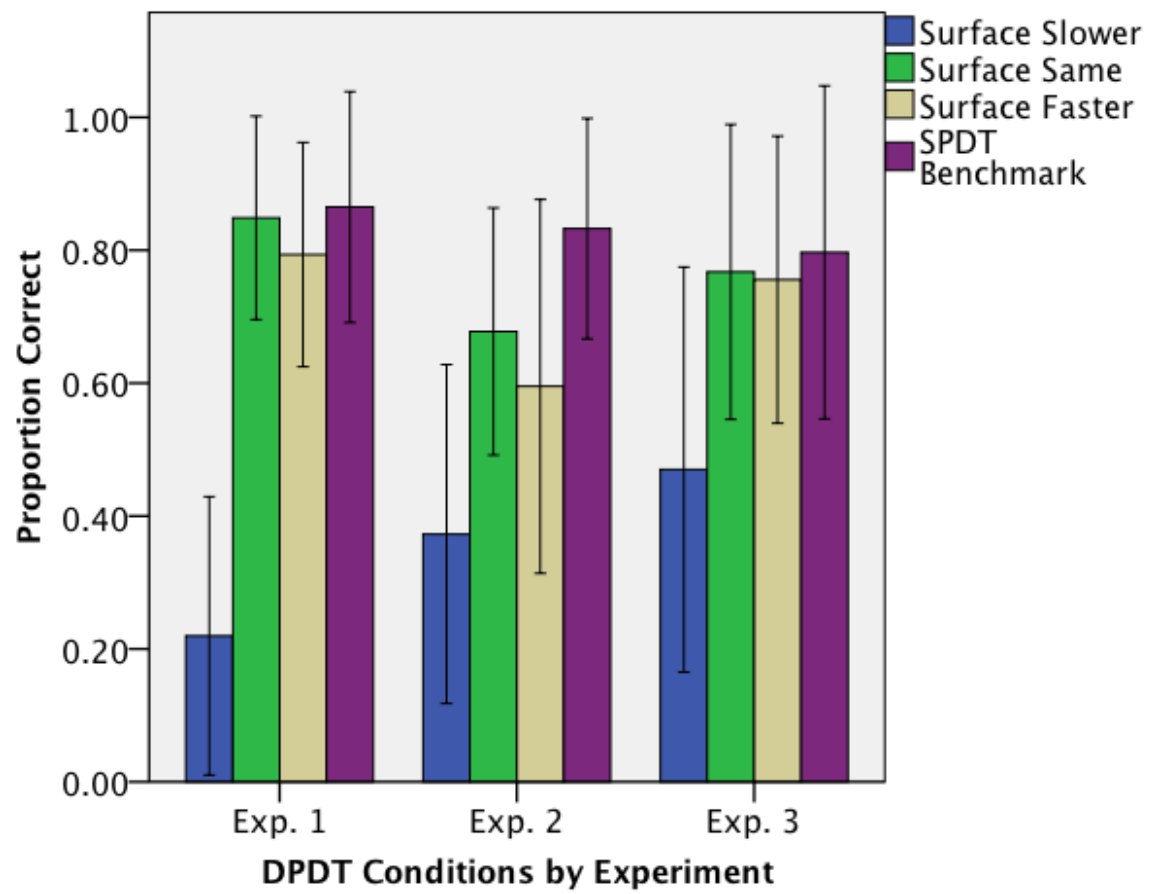

Error bars: +/- 1 SD

Figure 4. DPDT Condition, surface $\mathrm{x}$ beat rate, Experiments 1, 2, and 3.

\begin{tabular}{|l|c|c|c|c|c|c|}
\hline Experiment & \multicolumn{2}{|l|}{ Surface Slower } & \multicolumn{2}{l|}{ Surface Same } & \multicolumn{2}{l|}{ Surface Faster } \\
\hline & Mean & SD & Mean & SD & Mean & SD \\
\hline Exp 1 & 21.96 & 20.94 & 84.88 & 15.30 & 79.33 & 16.88 \\
\hline Exp 2 & 37.29 & 25.49 & 67.78 & 18.61 & 59.52 & 28.14 \\
\hline Exp 3 & 47.00 & 30.48 & 76.74 & 22.19 & 75.58 & 21.60 \\
\hline
\end{tabular}

Table 4. Percent correct and standard deviation of participant responses in each subset of DPDT Slow-Fast conditions.

Table 4 allows us to see how changing the experimental task affected participant accuracy and results. In the "Surface Slower" condition the mean percent correct steadily increases from Experiment 1 to Experiment 2 to Experiment 3. As is also clear in Table 4, the variance is also increasing; thus while the improvement from Experiment 1 to Experiment 2 is significant $(\mathrm{Z}=-2.318 p=.020$, Mann-Whitney $\mathrm{U}$ 
test), the improvement from Experiment 2 to Experiment 3 is not $(\mathrm{Z}=-.772, p=.440$, Mann-Whitney $\mathrm{U}$ test). Conversely, performance in the other two conditions ("Surface Same" and "Surface Faster") degrades from Experiment 1 to Experiments 2 and 3. Differences between Experiments 1 and 2 were highly significant (surface same $\mathrm{Z}=-2.501, p=.012$; surface faster $\mathrm{Z}=-2.305, p=.021$, Mann-Whitney U Test), but were not significant between Experiment 1 and Experiment 3 or between Experiment 2 and 3 .

Finally, in looking at the DPDT responses to the "mismatched" subset of both the Slow-Fast (SF) and Fast-Slow (FS) conditions, we find that when participants did give the "wrong" response, they overwhelmingly made their tempo judgments in accord with the surface rates. These responses are summarized in Table 5.

\begin{tabular}{|c|c|c|c|c|c|c|}
\hline Condition & \multicolumn{3}{|c|}{ DPDT SF } & \multicolumn{3}{c|}{ DPDT FS } \\
\hline Experiment & Exp1 & Exp2 & Exp3 & Exp1 & Exp2 & Exp3 \\
\hline "wrong" \% & 85.88 & 67.01 & 80.85 & 86.84 & 75.73 & 73.51 \\
\hline
\end{tabular}

Table 5. Percentage of the "wrong" responses in the mismatched SF and FS conditions that corresponded to the surface rates of motion.

\section{Tapping Bias Analysis}

The DPDT analysis in Experiments 2 and 3 shows the effect of focusing on the tactus level (either without or with overt tapping) on tempo judgments and it also raises a question: does the manner in which one taps bias one's tempo judgment in any way? An analysis of tapping data from the DPST condition in Experiment 3 allows a preliminary glimpse at the effect of tapping behavior on tempo judgment.

As noted in the introduction, at the tempos used in these experiments more than one beat percept may be possible and hence more than one mode of tapping to the beat may ensue. Indeed, this is what we found in an analysis of participant tapping. Tapping modes ranged from tapping every note (i.e., at the 250 $\mathrm{ms}$ or $300 \mathrm{~ms}$ inter-tap interval), every beat (500 ms or $600 \mathrm{~ms}$ ITI), every other beat (1000 ms or $1200 \mathrm{~ms}$ ITI), and for one participant, every downbeat (i.e., once per cycle, at $2000 \mathrm{~ms}$ or $2400 \mathrm{~ms}$ intervals). Most responses were at the beat level and most participants (14 of 19) tapped at the same level at least $66 \%$ of the time within and across all trials. The remaining 5 tended to switch between the beat and two-beat levels. Of the "stable" tappers, nine tapped at the beat rate (and very consistently, in $87 \%$ of all trials on average), and five tapped at slower rates (four at twice the beat rate and one at four times the beat rate; the very slow tapper was omitted from the analysis). This allowed us to group the participants according to their tapping mode as indicated in Figure 5.

In the DPST condition, as the standard and comparison have the same beat rate, the correct answer of the 3AFC should be "the same," and errors, if present, should be evenly distributed in the other two response categories. If a participant is biased, however, this will affect the distribution of the incorrect responses. In the analysis given in Figure 5 bias was calculated in the following manner. Responses were scored as $-1=$ slower, $0=$ same, and $1=$ faster and then averaged. In the DPST condition, a "perfect" score would be zero for all trials, and a pattern of unbiased errors would leave the mean near zero. As can be seen in Figure 5, the slower tappers tended to judge the target as slower (even though it was the same) while other tappers did not. The mean score for tactus tappers was .0097 , while the mean score for the $2 \mathrm{x}$ tactus tappers was -.0805. This difference is almost significant: $t(11)=2.178, p=.052$ (independent samples $t$ test, two-tailed); given the bias is in the direction one would predict (slower tapping yields slower tempo judgment) one may use a one-tailed test $(p=.026)$. Nonetheless, caution should be observed given the small sample sizes. But the implication is clear: our sense of musical tempo may come, at least in part, from the kind of self-motion the music engenders. 


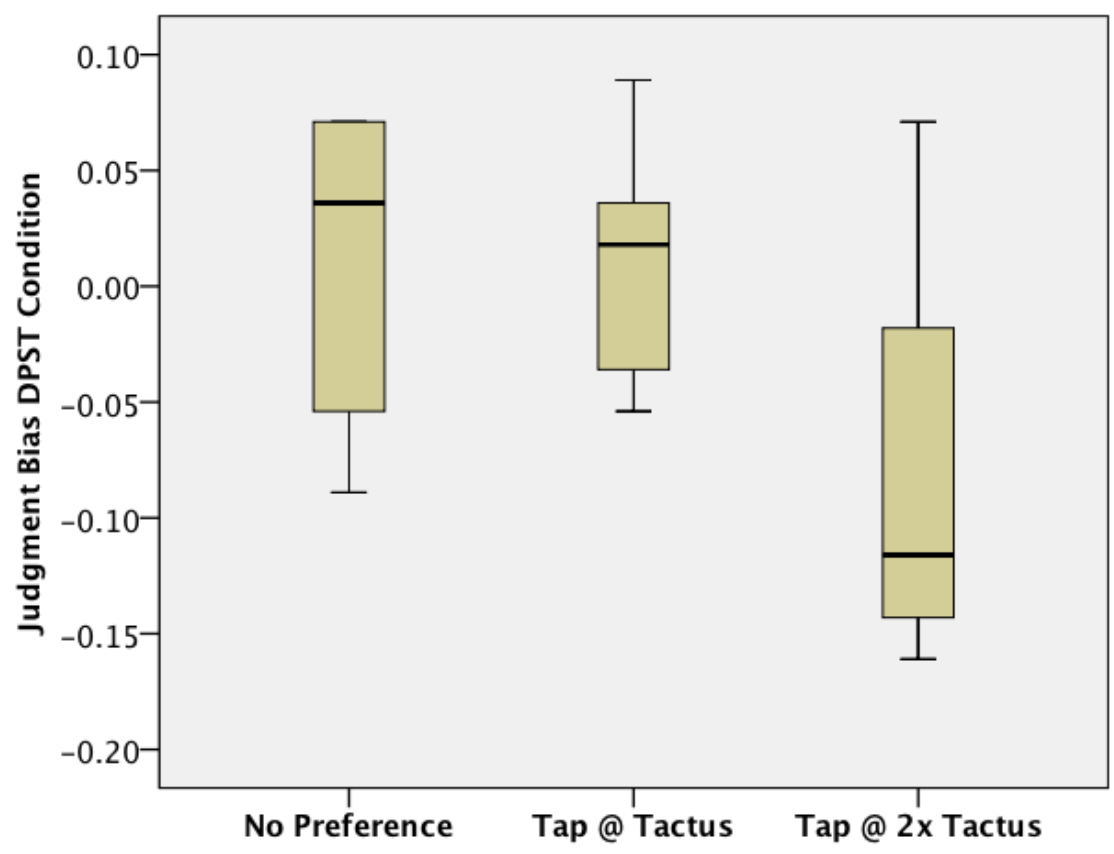

Figure 5. Boxplot of bias effect of tapping mode on tempo judgment, Experiment 3, DPST condition.

\section{DISCUSSION}

These experiments show that tempo judgment is more than a simple assessment of the tactus rate. Differences in metrical structure affect tempo judgments, even when the rates of surface activity are the same or commensurate, as evidenced by the main effects observed in Experiments 1 and 2. Analyses of the DPDT condition in all three experiments show that when there is a conflict or mismatch between tactus rate and surface rate, tempo judgments become more diffuse; in such cases surface density (i.e., the presence or absence of subdivision of the tactus) becomes more salient than the tactus rate. There may be an interesting parallel here with motion perception in vision. Verghese \& Stone (1996) have shown that the perception of speed in visual arrays depends on the coherence and segmentability of the elements in the array; they show that "the effect of both proximity and segmentation [of a visual array] on speed discrimination suggest that local speed mechanisms do not act in isolation, but rather in assemblies that have both neighboring and long-range interactions" (Verghese \& Stone, 1996, p. 163).

Experiments 2 and 3 show that focusing on and/or engaging with the tactus rate does not lead to a straightforward improvement in tempo judgment or greater inter-individual agreement (indeed, such agreement was lessened in these two experiments). While focusing on the beat rate did improve performance in the mismatched DPDT condition in Experiment 2 relative to Experiment 1, in the other DPDT conditions focusing on the beat actually reduced performance. Likewise tapping along did not give any significant benefit (Experiment 3 vs. Experiment 2, mismatched DPDT condition). This may be because the potential benefit of tapping (i.e., a robust motor engagement with the tactus level of the stimulus) also comes with some cognitive costs (i.e., the need to monitor one's sensorimotor coordination with the stimulus, correct phase and period errors, etc.).

Thus in terms of our initial hypotheses we can say that:

- Differences in surface rhythm and metrical structure do interfere with judgments of tempo, even if two passages have the same beat rate.

- Event density (i.e., subdivision) is more salient than tactus rate when there is a mismatch between beat and surface rates of activity. 
- Focusing on the tactus level does not improve the overall accuracy and consistency of tempo judgments; while it may improve performance in some conditions (DPDT), it may degrade it in others.

- Tapping along also does not lead to a further improvement in accuracy and consistency of tempo judgments.

More broadly, the interaction between tactus rate and the rhythmic surface is complex. While many studies of beat induction and tempo judgment have used isochronous and "stationary" (i.e., continuous and periodic) stimuli, in music and other rhythmic contexts, the stimuli are more variegated and complex. While this study did not examine the former, as would be the case when looking at stimuli involving a mixture of surface durations that nonetheless project a stable beat, it did examine the latter, looking at stimuli with the same or similar surface activity (IOI rates) but different organization at higher levels/periodicities.

Finally, the analysis of tapping mode bias (Experiment 3, DPST condition) shows that the manner/rate of sensorimotor coordination one has with a rhythmic pattern may affect one's sense of its speed. It is clear then, that judgments of musical tempo involve multiple components:

- Our sense of the rate(s) of motion "in the music";

- Our own rate of motion, and our sense of it;

- Finding/negotiating our interaction with the music via periodicity that affords a coordination between the two.

These conclusions suggest a number of lines for future research. The author and colleagues are currently engaged in tempo judgment research in which tapping mode is controlled and participants are presented with rhythmic and melodic stimuli in a broad range of tempos. Additional work is needed on basic tempo judgments using rhythmic and melodic stimuli more complex and ecologically valid than the simple "stationary" patterns used in the three experiments. Finally, as listening to musical and non-musical rhythms engages one's entire body, studies which involve more complex assays of sensorimotor engagement with rhythmic stimuli may shed additional light on how we grasp that "integrated bundle of musical elements which flows with a rightful sense."

\section{NOTES}

[1] A terminological clarification. The musical beat is also referred to as the pulse or tactus (Lerdahl \& Jackendof, 1983; London, 2001; Martens, 2005); the terms tactus and beat will be used here interchangeably to refer to the regular level of rhythmic activity in the musical texture with an IOI in the range of approximately 500-1500 ms that (a) gives rise to a strong sense of sensorimotor synchronization, (b) can be further subdivided into shorter units that still have a sense of rhythmic identity (i.e., having an IOI greater than $100 \mathrm{~ms}$ ), and (c) can be linked into longer cycles comprised of 2-7 beats.

[2] Here and below non-parametric tests (i.e., Wilcoxon Signed Ranks or Mann-Whitney U) were used for comparisons when the data did not follow a normal distribution, which was the case most of the time; analogous parametric tests were used when appropriate. In all tests $p$ values are two-tailed unless otherwise indicated.

\section{REFERENCES}

Clarke, E. (1999). Rhythm and timing in music. In: D. Deutsch (Ed.), The Psychology of Music. New York: Academic Press, pp. 473-500.

Clynes, M., \& Walker, J. (1982). Neurobiologic functions of rhythm, time, and pulse in music. In: M. Clynes (Ed.), Music, Mind, and Brain. New York: Plenum Press, pp. 171-216.

Collyer, C.E., Broadbent, H.A., \& Church, R.M. (1994). Preferred rates of repetitive tapping and 
categorical time production. Perception and Psychophysics, Vol. 55, No. 4, pp. 443-453.

Drake, C., Gros, L., \& Penel, A. (1999). How fast is that music? The relation between physical and perceived temp. In: S. W. Yi (Ed.), Music, Mind, and Science. Seoul: Seoul National University, pp. 190203.

Drake, C., Penel, A., \& Bigand, E. (2000). Why musicians tap slower than non-musicians. In: P. Desain \& W.L. Windsor (Eds.), Rhythm Perception and Production. Lisse: Swets \& Zeitlinger, pp. 245-248.

Eck, D. (2001). A positive-evidence model for rhythmical beat induction. Journal of New Music Research, Vol. 30, No. 2, pp. 187-200.

Epstein, D. (1979). Beyond Orpheus. Cambridge: MIT Press.

Epstein, D. (1995). Shaping Time: Music, the Brain, and Performance. New York: Schirmer.

Fraisse, P. (1982). Rhythm and tempo. In: D. Deutsch. (Ed.), The Psychology of Music. New York: Academic Press, pp. 149-180.

Jones, M.R., \& Boltz, M. (1989). Dynamic attending and responses to time. Psychological Review, Vol. 96, No. 3, pp. 459-91.

Large, E.W., Fink, P., \& Kelso, S. (2002). Tracking simple and complex sequences. Psychological Research, Vol. 66, pp. 3-17.

Lerdahl, F., \& Jackendoff, R. (1983). A Generative Theory of Tonal Music. Cambridge: MIT Press.

London, J. (2001). Rhythm. In: S. Sadie \& J. Tyrrell, (Eds.), The New Grove Dictionary of Music and Musicians, 2nd Edition. New York: Grove Publishing, Vol. 21, pp. 277-309.

London, J. (2004). Hearing in Time. New York: Oxford University Press.

Martens, P.A. (2005). Beat-finding, listener strategies, and musical meter. Ph.D. dissertation, Department of Music, University of Chicago.

McKinney, M.F., \& Moelants, D. (2006). Ambiguity in tempo perception: What draws listeners to different metrical levels? Music Perception, Vol. 24, No. 2, pp. 155-165.

Moelants, D. (2006). Perception and performance of aksak metres. Musicae Scientiae, Vol. 10, No. 2, pp. 147-172.

Parncutt, R. (1994). A perceptual model of pulse salience and metrical accent in musical rhythms. Music Perception, Vol. 11, No. 4, pp. 409-464.

Quinn, S., \& Watt, R. (2006). The perception of tempo in music. Perception, Vol. 35, No. 2, pp. 267-280.

Repp, B.H. (2005). Sensorimotor synchronization: A review of the tapping literature. Psychonomic Bulletin \& Review, Vol. 12, No. 6, pp. 969-992.

Repp, B.H. (2007). Hearing a melody in different ways: Multistability of metrical interpretation, reflected in rate limits of sensorimotor synchronization. Cognition, Vol. 102, No. 3, pp. 434-454.

Snyder, J., \& Krumhansl, C.L. (2001). Tapping to ragtime: Cues to pulsefinding. Music Perception, Vol. 18, No. 4, pp. 455-489. 
Thayer, A.W., \& Harvey, D. (2010). Maelzel, Johann Nepomuk. Grove Music Online. Oxford Music Online, http://www.oxfordmusiconline.com/subscriber/article/ grove/music/17414. Accessed 16 Aug. 2010 .

van Noorden, L., \& Moelants, D. (1999). Resonance in the perception of musical pulse. Journal of New Music Research, Vol. 28, No. 1, pp. 43-66.

Verghese, P., \& Stone, L.S. (1996). Perceived visual speed constrained by image segmentation. Nature, Vol. 381, No. 9, pp. 161-163. 